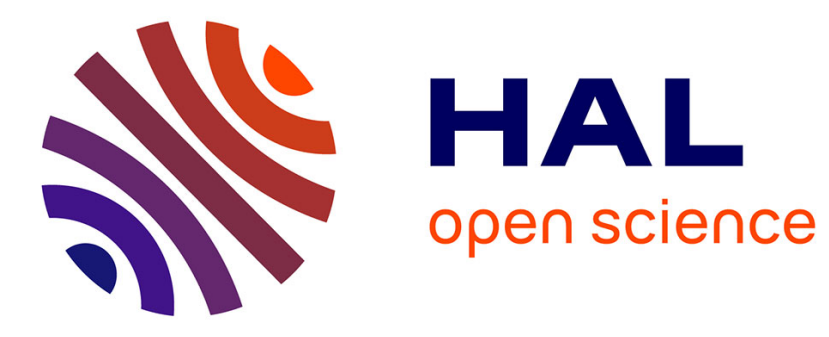

\title{
2-distance coloring of sparse graphs
}

\author{
Marthe Bonamy, Benjamin Lévêque, Alexandre Pinlou
}

\section{To cite this version:}

Marthe Bonamy, Benjamin Lévêque, Alexandre Pinlou. 2-distance coloring of sparse graphs. Eurocomb'11: European Conference on Combinatorics, Graph Theory and Applications, Aug 2011, Budapest, Hungary. pp.155-160, 10.1016/j.endm.2011.09.027 · lirmm-00782852

\section{HAL Id: lirmm-00782852 https://hal-lirmm.ccsd.cnrs.fr/lirmm-00782852}

Submitted on 30 Jan 2013

HAL is a multi-disciplinary open access archive for the deposit and dissemination of scientific research documents, whether they are published or not. The documents may come from teaching and research institutions in France or abroad, or from public or private research centers.
L'archive ouverte pluridisciplinaire HAL, est destinée au dépôt et à la diffusion de documents scientifiques de niveau recherche, publiés ou non, émanant des établissements d'enseignement et de recherche français ou étrangers, des laboratoires publics ou privés. 


\title{
2-distance coloring of sparse graphs *
}

\author{
Marthe Bonamy ${ }^{\mathrm{a}, 1}$ Benjamin Lévêque ${ }^{\mathrm{a}}$ Alexandre Pinlou ${ }^{\mathrm{a}, 2}$ \\ a LIRMM, Université Montpellier 2, CNRS \\ \{marthe.bonamy, benjamin. leveque, alexandre.pinlou\}@lirmm.fr
}

\begin{abstract}
A 2-distance coloring of a graph is a coloring of the vertices such that two vertices at distance at most 2 receive distinct colors. We prove that every graph with maximum degree $\Delta$ at least 4 and maximum average degree less that $\frac{7}{3}$ admits a 2 -distance $(\Delta+1)$-coloring. This result is tight. This improves previous known results of Dolama and Sopena.
\end{abstract}

Keywords: 2-distance coloring; square coloring; maximum average degree.

\section{Introduction}

All the graphs we consider here are simple, finite and undirected. Let $G=$ $(V, E)$ be a graph. For any subgraph $H$ of $G$, we denote $V(H)$ and $E(H)$ the vertices and edges of $H$. For any vertex $v \in V$, the degree of $v$ in $G$, denoted $d(v)$, is the number of neighbors of $v$ in $G$. The maximum degree of $G$, denoted $\Delta(G)$, is $\max _{v \in V} d(v)$. The maximum average degree of $G$, denoted $\operatorname{mad}(G)$, is the maximum for every subgraph $H$ of $G$ of $\frac{2|E(H)|}{|V(H)|}$. A 2-distance coloring

ऋ Research partially supported by the ANR GRATOS, contract ANR-09-JCJC-0041-01.

1 École Normale Supérieure de Lyon

2 Second affiliation: Département MIAp, Université Paul-Valéry, Montpellier 3 
of a graph $G$ is a coloring of the vertices of $G$ such that two vertices that are adjacent or have a common neighbor receive distinct colors. This is equivalent to a proper vertex-coloring of the square of $G$. We define $\chi^{2}(G)$ as the smallest $k$ such that $G$ admits a 2 -distance $k$-coloring. Note that any graph $G$ satisfies $\chi^{2}(G) \geq \Delta(G)+1$. The girth $g(G)$ is the length of a shortest cycle in $G$. Two vertices $x$ and $y$ are $p$-linked if there exists a path $x-v_{1}-\cdots-v_{p}-y$ such that vertices $v_{1}, \ldots, v_{p}$ have degree 2 , and $v_{1}-\cdots-v_{p}$ is called a branch of $x$ (or $y$ ).

Borodin, Ivanova and Neustroeva [1] studied sparse planar graphs, and prove the following result:

Theorem 1.1 ([1]) Every planar graph $G$ with $g(G) \geq 15$ and $\Delta(G) \geq 4$ admits a 2-distance $(\Delta(G)+1)$-coloring.

Note that this result was later extended to list-coloring [2].

Dolama and Sopena [3] proved a more general result than Theorem 1.1, which is not restricted to planar graphs anymore. Theorem 1.2 however presents a slight loss in quality compared to Theorem 1.1: since for any planar graph $G,(\operatorname{mad}(G)-2)(g(G)-2)<4$, Theorem 1.2 implies only that Theorem 1.1 holds for $g(G) \geq 16$.

Theorem $1.2([3])$ Every graph $G$ with $\operatorname{mad}(G)<\frac{16}{7}$ and $\Delta(G) \geq 4$ admits a 2-distance $(\Delta(G)+1)$-coloring.

We aim at making the upper bound on the maximum average degree optimal, and prove the following.

Theorem 1.3 Every graph $G$ with $\operatorname{mad}(G)<\frac{7}{3}$ and $\Delta(G) \geq 4$ admits a 2-distance $(\Delta(G)+1)$-coloring.

The bound we obtain is optimal. Indeed, as pointed out by Montassier [6], there is a graph $G$ with $\operatorname{mad}(G)=\frac{7}{3}, \Delta(G)=4$ and $\chi^{2}(G)=6$ (see Figure 1 ).

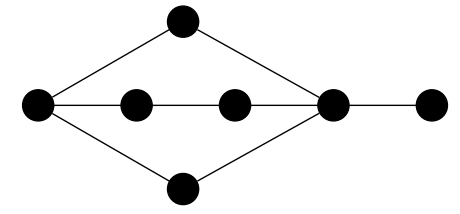

Fig. 1. A graph $G$ with $\operatorname{mad}(G)=\frac{7}{3}, \Delta(G)=4$ and $\chi^{2}(G)=6$.

When restricted to planar graphs, Theorem 1.3 is an improvement of Theorem 1.1 as it implies that Theorem 1.1 holds with $g(G) \geq 14$. It is not comparable to the more general result in [2], since we are not considering list-coloring. 
We are going to use a discharging method to prove Theorem 1.3. We will prove that there are some configurations a minimal counter-example cannot contain, and, then use discharging rules to show that this graph does not exist.

\section{Proof}

In the figures, we draw in black a vertex that has no other neighbor than the ones already represented, in white a vertex that might have other neighbors than the ones represented. When there is a label inside a white vertex, it is an indication on the number of neighbors it has. The label ' $i$ ' means "exactly $i$ neighbors", the label ' $i^{+}$' (resp. ' $i^{-}$') means that it has at least (resp. at most) $i$ neighbors. Note that the white vertices may coincide with other vertices. The label ' $T(v, a)$ ' inside a vertex $v$ means that $T(v, a)$ exists, as defined below.

A configuration $T\left(v, a_{4}\right)$ (see Figure 2 ), is inductively defined as a vertex $v$ of degree 4 with neighbors $a_{1}, a_{2}, a_{3}, a_{4}$, where for $i \in\{1,2,3\}$, vertex $v$ is 2-linked by a path $v-a_{i}-b_{i}-w_{i}$ either to a vertex $w_{i}$ of degree at most 3 or to a configuration $T\left(w_{i}, b_{i}\right)$.

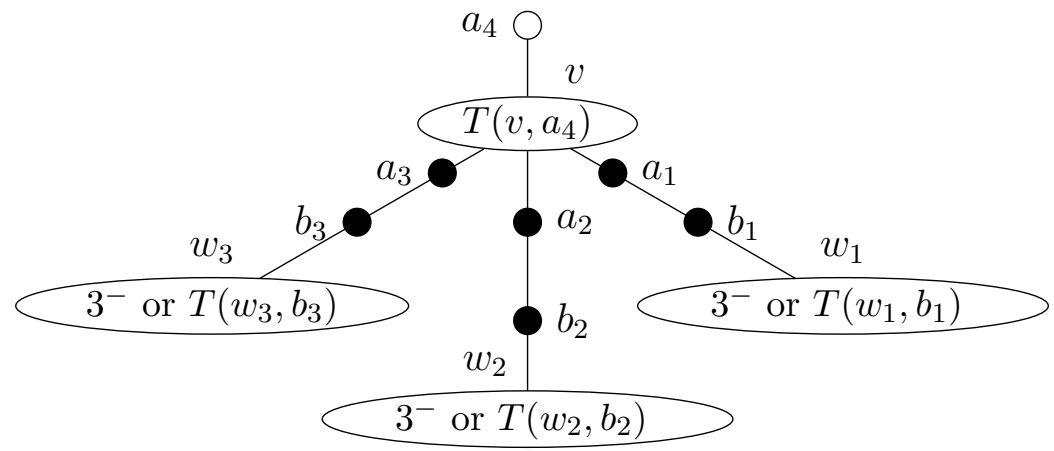

Fig. 2. A $T\left(v, u_{4}\right)$.

Now we define configurations $\left(C_{1}\right)$ to $\left(C_{5}\right)$ (see Figure 3 ).

- $\left(C_{1}\right)$ is a vertex of degree 0 or 1.

- $\left(C_{2}\right)$ is a vertex 3-linked to a vertex not of maximal degree.

- $\left(C_{3}\right)$ is a vertex of degree 3 that is 2 -linked to two vertices of degree 3 , and 1-linked to a vertex of degree at most 3.

- $\left(C_{4}\right)$ is a vertex $u$ of degree at most 3 that is 2 -linked by a path $u-y-x-v$ to a vertex $v$ such that $T(v, x)$ exists.

- $\left(C_{5}\right)$ is a vertex $u$ of degree 3 that is 2-linked to two vertices, and 1-linked by a path $u-x-v$ to a vertex $v$ such that $T(v, x)$ exists. 

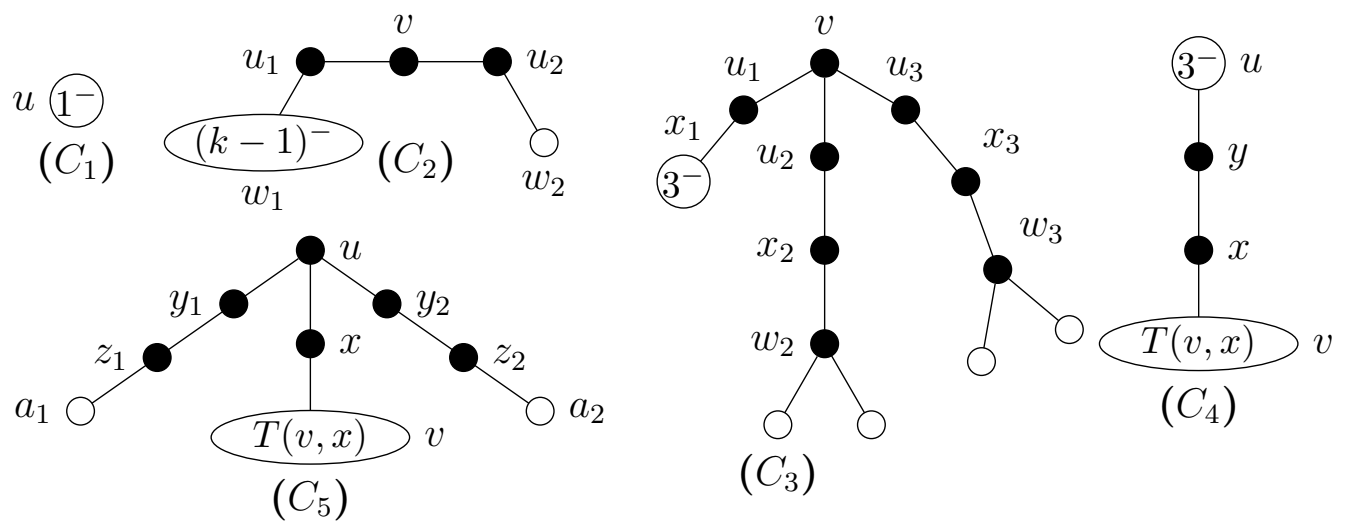

Fig. 3. Forbidden configurations.

In the following lemma, we actually use $k$ instead of $\Delta(G)$ in order to ensure that any subgraph of $G$ admits a $(k+1)$-coloring even though $\Delta$ can decrease.

A graph is minimal for a property if it satisfies this property but none of its subgraphs does.

Lemma 2.1 Let $k \geq 4$ and $G$ such that $\Delta(G) \leq k$ and $G$ admits no 2-distance $(k+1)$-coloring, and $G$ is minimal for this property. Then $G$ does not contain any of Configurations $\left(C_{1}\right)$ to $\left(C_{5}\right)$.

The following lemma will ensure that the discharging rules we introduce later are well-defined.

Lemma 2.2 In a graph $G$ where $\left(C_{4}\right)$ is forbidden, and $x$ and $y$ are two vertices of degree 4 that are 2 -linked by a path $x$-a-b-y, at most one of $T(x, a)$ and $T(y, b)$ exists.

We design discharging rules $R_{1}, R_{2}, R_{3}$ (see Figure 4 ). We use them in the proof of Lemma 2.3, where the initial weight of a vertex equals its degree, and its final weight is shown to be at least $\frac{7}{3}$. For any two vertices $x$ and $y$ of degree at least 3 , with $d(x) \geq d(y)$,

- Rule $R_{1}$ is when $x$ and $y$ are 1 -linked by a path $x-a-y$.

- $\left(R_{1.1}\right)$ If $d(x)=d(y)$, then both $x$ and $y$ give $\frac{1}{6}$ to $a$.

- $\left(R_{1.2}\right)$ If $d(x)>d(y)$ and $T(x, a)$ exists, then both $x$ and $y$ give $\frac{1}{6}$ to $a$.

- $\left(R_{1.3}\right)$ If $d(x)>d(y)$ and $T(x, a)$ does not exist, then $x$ gives $\frac{1}{3}$ to $a$.

- Rule $R_{2}$ is when $x$ and $y$ are 2-linked by a path $x-a-b-y$.

- $\left(R_{2.1}\right)$ If $d(x)=d(y)$ and neither $T(x, a)$ nor $T(y, b)$ exist, then $x$ (resp. $y$ ) gives $\frac{1}{3}$ to $a$ (resp. $b$ ). 
- $\left(R_{2.2}\right)$ If $d(x)=d(y)$ and $T(y, b)$ exists, then $x$ gives $\frac{1}{3}$ to $a$ and both $x$ and $y$ give $\frac{1}{6}$ to $b$.

- $\left(R_{2.3}\right)$ If $d(x)>d(y)$, then $x$ gives $\frac{1}{3}$ to $a$ and both $x$ and $y$ give $\frac{1}{6}$ to $b$.

- Rule $R_{3}$ is when $x$ and $y$, both of degree at least 4, are 3-linked by a path $x-a-b-c-y$. Then $x$ gives $\frac{1}{3}$ to $a$ and $\frac{1}{6}$ to $b$, and symmetrically for $y$.

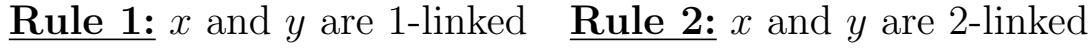

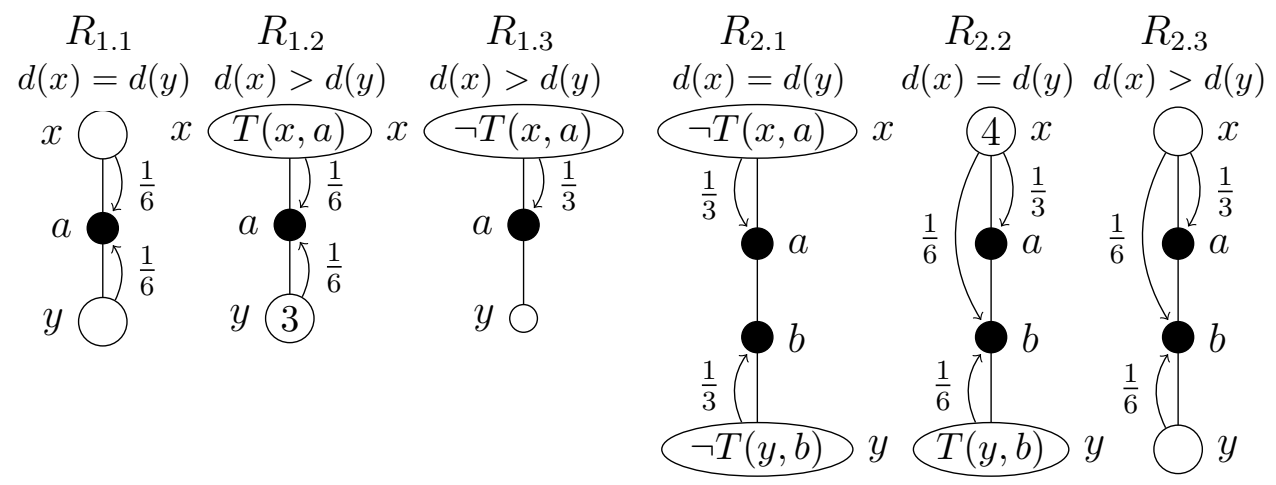

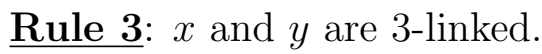

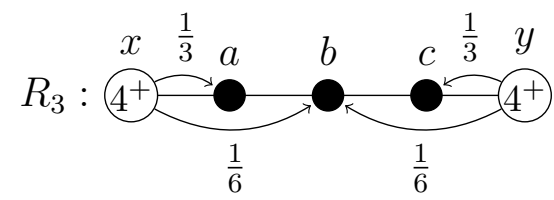

Fig. 4. Discharging rules $R_{1}, R_{2}, R_{3}$.

We use these discharging rules to prove the following lemma:

Lemma 2.3 A graph $G$ that does not contain Configurations $\left(C_{1}\right)$ to $\left(C_{5}\right)$ verifies $\operatorname{mad}(G) \geq \frac{7}{3}$.

\section{Proof of Theorem 1.3}

We prove a stronger version of Theorem 1.3 by contradiction. For $k \geq 4$, let $G$ be a minimal graph such that $\Delta(G) \leq k, \operatorname{mad}(G)<\frac{7}{3}$ and $G$ does not admit a $(k+1)$-coloring. Graph $G$ is also a minimal graph such that $\Delta(G) \leq k$ and $G$ does not admit a $(k+1)$-coloring (all its proper subgraphs verify $\Delta \leq k$ and mad $<\frac{7}{3}$, so they admit a $(k+1)$-coloring). By Lemma 2.1, graph $G$ cannot contain $\left(C_{1}\right)$ to $\left(C_{5}\right)$. Lemma 2.3 implies that $\operatorname{mad}(G) \geq \frac{7}{3}$. Contradiction. 


\section{Conclusion}

We actually proved a slightly stronger result than Theorem 1.3. However, the addition, namely that every graph $G$ with $\operatorname{mad}(G)<\frac{7}{3}$ and $\Delta(G) \leq 3$ admits a 2-distance 5-coloring, can be derived from a result of Dvořák, Škrekovski and Tancer [4].

Note that the proof of Theorem 1.3 also provides an $O\left(|V|^{3}\right)$ algorithm to find a 2-distance coloring of a graph $G$ with $\Delta(G)+1$ colors if $G$ verifies the hypothesis of Theorem 1.3: indeed Lemma 2.3 proves that every graph $G$ with $\operatorname{mad}(G)<\frac{7}{3}$ contains $\left(C_{1}\right),\left(C_{2}\right), \ldots$ or $\left(C_{5}\right)$. Consequently, we can find a $\left(C_{i}\right)$ in $G$, remove the corresponding vertices, and extend the coloring to the initial graph using the proof of Lemma 2.1.

As it was conjectured by Kostochka and Woodall [5] that 2-distance listcoloring requires exactly as many colors as 2-distance coloring, future work could aim at extending Theorem 1.3 to list-coloring.

\section{References}

[1] Borodin, O. V., A. O. Ivanova and N. T. Neustroeva, 2-distance coloring of sparse plane graphs (in russian), Siberian Electronic Mathematical Reports 1 (2004), pp. $76-90$.

[2] Borodin, O. V., A. O. Ivanova and N. T. Neustroeva, List 2-distance $(\Delta+1)$ coloring of planar graphs with given girth, Journal of Applied and Industrial Mathematics 2 (2008), pp. 317-328.

[3] Dolama, M. H. and É. Sopena, On the maximum average degree and the incidence chromatic number of a graph, Discrete Mathematics and Theoretical Computer Science 7 (2005), pp. 203-216.

[4] Dvořák, Z., R. Škrekovski and M. Tancer, List-Coloring squares of sparse subcubic graphs, SIAM Journal on Discrete Mathematics 22 (2008), pp. 139159 .

[5] Kotsochka, A. V., and D. R. Woodall, Choosability conjectures and multicircuits, Discrete Mathematics 240 (2001), pp. 123-143.

[6] Montassier, M., Personal Communication. 